\title{
Modulated spin structure responsible for the magnetic-field-induced polarization switching in multiferroic $\mathrm{TbMn}_{2} \mathrm{O}_{5}$
}

\author{
Jung-Hoon Lee ${ }^{1, *}$ and Hyun Myung Jang ${ }^{1,2, \dagger}$ \\ ${ }^{1}$ Department of Materials Science and Engineering, and Division of Advanced Materials Science (AMS), Pohang University of Science and \\ Technology (POSTECH), Pohang 790-784, Republic of Korea \\ ${ }^{2}$ Department of Physics, Pohang University of Science and Technology (POSTECH), Pohang 790-784, Republic of Korea
}

(Received 20 October 2014; published 5 January 2015)

\begin{abstract}
Orthorhombic $\mathrm{TbMn}_{2} \mathrm{O}_{5}(o-\mathrm{TMO})$ is a well-known multiferroic manganite with the remarkable property of polarization switching at $3 \mathrm{~K}$ under a bias magnetic $(H)$ field along the $a$ axis of $P b 2_{1} \mathrm{~m}$. To theoretically account for this outstanding observation, we have proposed a modulated spin structure under the saturated bias $H$ field by considering the relative strength of the three relevant exchange parameters in $o$-TMO. The proposed modulated structure based on density-functional theory (DFT) calculations is described in terms of the spin angle $\phi$ between the neighboring $\mathrm{Mn}^{4+}-\mathrm{Mn}^{3+}$ spin moments on the $a-b$ plane. We have shown that the computed DFT polarization plotted as a function of $\phi$ satisfactorily accounts for the observed $H$-field-induced polarization switching. We have further theoretically shown that the square of the critical field strength $\left(H_{c}\right)$ needed for the polarization switching is inversely proportional to the degree of the extrinsic magnetoelectric coupling. The computed partial charge density demonstrates that the $H$-field-induced polarization switching also accompanies with the switching in the sign of the excess valence-electron density.
\end{abstract}

DOI: 10.1103/PhysRevB.91.014403

PACS number(s): 75.85.+t, 71.15.Mb, 75.50.Ee, 77.84.-s

\section{INTRODUCTION}

Over the past decade, there has been a resurgence of interest in understanding and technological applications of multiferroics [1-6]. These materials have received a great deal of attention owing to their potential for enabling new device paradigms that are based on the cross coupling between distinct order parameters [2-6]. Among numerous multiferroics currently under investigation, manganite-based oxides have been most extensively studied by virtue of their strong tendency of the magnetoelectric (ME) coupling [2,3,7-9]. One of the most prominent examples of manganite-based multiferroics is orthorhombic $\mathrm{TbMn}_{2} \mathrm{O}_{5}$ (o-TMO) $[3,10]$. Currently, the most outstanding feature of $o$-TMO is the polarization switching at $3 \mathrm{~K}$ under a bias magnetic $(H)$ field along the $a$ axis of $P b 2_{1} m$ [3]. $o$-TMO is characterized by the complex periodicity in the temperature-dependent spin structure [10]. An incommensurate antiferromagnetic (AFM) ordered state appears below $43 \mathrm{~K}$ with the associated ferroelectricity at $38 \mathrm{~K}$. With decreasing temperature, a propagation vector $\boldsymbol{k}$ then locks into a commensurate value of $(0.5,0,0.25)$ at $33 \mathrm{~K}$. At $24 \mathrm{~K}$, the propagation vector $\boldsymbol{k}$ surprisingly becomes incommensurate again. In the temperature region between 33 and $24 \mathrm{~K}$, the commensurate spin-induced ferroelectric polarization reaches its maximum value. Below $24 \mathrm{~K}, o$-TMO is again characterized by the incommensurate AFM structure with the propagation vector $\boldsymbol{k}$ of $(0.48,0,0.32)$ [10].

Following the discovery of the magnetic-field-induced polarization switching at $3 \mathrm{~K}$ by Hur et al. [3], many research groups have investigated the magnetic structure and the incommensurate AFM-ordering-induced ferroelectricity in $o$-TMO. Chapon et al. [10]. reported the magnetostructural phase diagram of $o$-TMO as a function of temperature and

\footnotetext{
*jhlee.david@gmail.com

†hmjang@postech.ac.kr
}

magnetic field by using neutron diffraction measurements. The ground-state structural, electronic, and magnetic properties of $o$-TMO were subsequently investigated by employing first-principles density-functional theory (DFT) calculations [11-14]. These ab initio studies confirmed that the groundstate structural symmetry of $o$-TMO is $P b 2_{1} m$ and the ferroelectricity in $o$-TMO is driven by the nearly collinear spin ordering that breaks the centrosymmetry without invoking the spin-orbit coupling. In spite of extensive experimental and computational studies on $o$-TMO, however, little progress has been made in our understanding of the modulated spin structures directly responsible for the magnetic-field-induced polarization-switching process which can be regarded as the single most important observation in multiferroics [3]. Accordingly, the main purpose of the present study is to computationally elucidate the modulation of the spin structure which is directly responsible for the observed polarization switching by a bias magnetic field at $3 \mathrm{~K}$.

\section{COMPUTATIONAL DETAILS}

To quantitatively understand the atomic-scale origin of the magnetic-field-induced polarization switching, we have performed DFT calculations on the basis of the generalized gradient approximation (GGA) and the GGA $+U$ method implemented in the projector augmented wave (PAW) [15] pseudopotential using the Vienna ab initio Simulation Package (VASP) [16]. To assess the effect of the spin-orbit coupling on the polarization switching, we have calculated the polarization by employing the Berry-phase method [17] with and without invoking the spin-orbit coupling. All the DFT calculations are performed using (i) a $450-\mathrm{eV}$ plane-wave cutoff energy and (ii) the tetrahedron method with the Blöchl corrections for the Brillouin zone integrations [18]. The Hubbard $U$ of $6.0 \mathrm{eV}$ and the exchange parameter $J$ of $0.8 \mathrm{eV}$ for Mn $3 d$ states are chosen on the basis of previous studies on $\mathrm{TbMn}_{2} \mathrm{O}_{5}$ [14] 

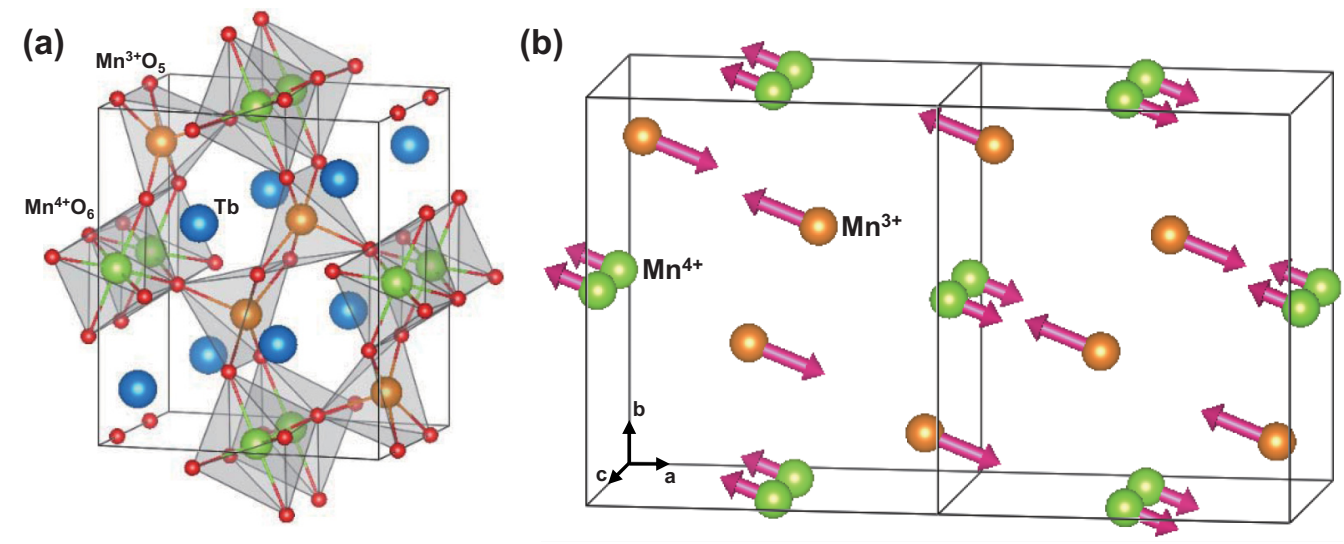

FIG. 1. (Color online) Crystal and spin structures of orthorhombic $\mathrm{TbMn}_{2} \mathrm{O}_{5}$ (o-TMO). (a) The unit-cell crystal structure of $o$-TMO in Pbam setting. (b) The ground-state spin configuration (+P structure) of $o$-TMO. Pink arrows denote the spin moments of Mn ions, and the orange and green balls represent $\mathrm{Mn}^{3+}$ and $\mathrm{Mn}^{4+}$ ions, respectively.

and $\mathrm{HoMn}_{2} \mathrm{O}_{5}$ [19]. We have explicitly treated nine valence electrons for $\operatorname{Tb}\left(5 p^{6} 5 d^{1} 6 s^{2}\right)$, seven for $\operatorname{Mn}\left(3 d^{5} 4 s^{2}\right)$, and six for $\mathrm{O}\left(2 s^{2} 2 p^{4}\right)$. Tb $4 f$ electrons are treated as a frozen core. We adopted a $2 a \times b \times c$ supercell ( 8 formula units) that imitates the propagation vector $\boldsymbol{k}=(0.5,0,0)$. This supercell had been used in the previous ab initio studies on $o$-TMO $[11,12,14]$. The validity of this approximation was justified in the previous work [11]. Thus, a $2 \times 4 \times 6$ Monkhorst-Pack $k$-points mesh centered at $\Gamma$ is used in actual calculations. The structural optimizations are performed for the 64-atoms cell until the forces on the relaxed cells are less than $0.005 \mathrm{eV} / \AA$. The lattice parameters used in our calculations are taken from the experimental results [20].

\section{RESULTS AND DISCUSSION}

The unit cell of $o$-TMO having four formula units (with 32 atoms) is composed of alternating $\mathrm{MnO}_{6}$ octahedron and $\mathrm{MnO}_{5}$ pyramid units, as shown in Fig. 1(a). The Mn ion located at the center of the octahedron unit is tetravalent $(4+)$ while the $\mathrm{Mn}$ ion located at the center of the pyramid unit is trivalent $(3+)$. The spin configuration of the antiferromagnetically ordered $o-\mathrm{TMO}$, as experimentally deduced by Chapon et al. [10], is schematically depicted in Fig. 1(b) and is denoted by " $+\mathrm{P}$ " structure. This configuration allows the electric polarization to develop along the $b$ axis in the $P b 2_{1} m$ space-group setting. Thus, the plus sign of $+\mathrm{P}$ signifies that the polarization direction is parallel to the $(+) b$ axis. According to our DFT calculations, the ferroelectric ground state is represented by this $+\mathrm{P}$ structure which breaks spatial inversion symmetry. Below $43 \mathrm{~K}$, the centrosymmetric Pbam structure is transformed to this polar $P b 2_{1} m$ structure by the incommensurate AFM spin ordering.

It is well known that there is a large discrepancy in the spontaneous polarization $\left(P_{s}\right)$ between the experimental and calculated values. In fact, the measured polarization of $o$-TMO is $\sim 45 \mathrm{nC} / \mathrm{cm}^{2}$ at $3 \mathrm{~K}[3,10]$. On the contrary, the calculated Berry-phase polarization without invoking the spinorbit interaction is remarkably larger than this value $[11,12]$. Most recently, Chang et al. [14]. significantly improved the computed $P_{s}$ value by adopting a high $U$ value. The
Berry-phase calculation is usually sensitive to the on-site repulsive Hubbard parameter $U$ value since this parameter makes the virtual electron hopping less favorable. Thus, the calculated $P_{s}$ significantly decreases with increasing $U$ value [19]. According to our Berry-phase calculations, the computed polarization with the $U$ value of $6.0 \mathrm{eV}$ is $75 \mathrm{nC} / \mathrm{cm}^{2}$ along the $b$ axis. This $P_{s}$ value is in good agreement with the value computed by Chang et al. [14]. and is the same order of magnitude as the experimentally reported $P_{s}$ value $[3,10]$. The computed $P_{s}$ value obtained by including the spinorbit interaction is $74 \mathrm{nC} / \mathrm{cm}^{2}$ which is nearly the same as that obtained without invoking the spin-orbit coupling $\left(75 \mathrm{nC} / \mathrm{cm}^{2}\right)$. This indicates that the inverse DzyaloshinskiiMoriya (DM) interaction, $\sim \boldsymbol{e}_{i j} \times\left(\boldsymbol{S}_{\boldsymbol{i}} \times \boldsymbol{S}_{j}\right)$, contributes only $1 \mathrm{nC} / \mathrm{cm}^{2}(<1 \%)$ to the net polarization. Thus, the exchangestriction mechanism of a $\left(S_{i} \cdot S_{j}\right)$ type is the origin of the magnetically induced polarization in $o$-TMO [11-14].

The appearance of the doubly degenerate polarization states by the exchange-striction mechanism can be quantitatively understood by considering the following free-energy density: $\Phi_{e}= \pm \gamma_{i}\left(S_{i}^{o} S_{j}^{o}\right) P+P^{2} / 2 \varepsilon_{o}$, where $\gamma_{i}$ is the intrinsic ME coupling constant; $S_{j}^{o}$ denotes a spin-order parameter and can be viewed as the amplitude of $\boldsymbol{S}_{\boldsymbol{j}}$, namely, $\left|\boldsymbol{S}_{\boldsymbol{j}}\right|=S_{j}^{o} e^{i \theta_{j}}$. The first term of the above equation denotes the coupling between the exchange-striction and the improper polarization. Minimizing $\Phi_{e}$ with respect to $P$ then yields the equilibrium improper polarization, $\pm P=\mp \gamma_{i} \varepsilon_{o}\left(S_{i}^{o} S_{j}^{o}\right)$. In the case of $o$-TMO, the polarization vector develops along the $b$ axis. Let us now examine the two spin configurations that are responsible for the two minima $(+\mathrm{P}$ and $-\mathrm{P})$ in the ferroelectric double-well potential. Here, both $+\mathrm{P}$ and $-\mathrm{P}$ structures should be represented by the same Kohn-Sham energy and the magnitude of polarization. The two improper polarization states can be switched to each other by applying an external electric field along the polar axis.

Figures 2(a) and 2(b) depict the spin configurations (upper panel) and the atomic displacement vectors (lower panel) that correspond to $+\mathrm{P}$ and $-\mathrm{P}$ structures, respectively. As can be deduced from Fig. 2(a), the net direction of the atomic displacement (ADP) of $\mathrm{Mn}^{3+}$ ions in the $+\mathrm{P}$ structure is along (+) $b$ axis which is parallel to the direction of $P_{s}$ in $o$-TMO. 

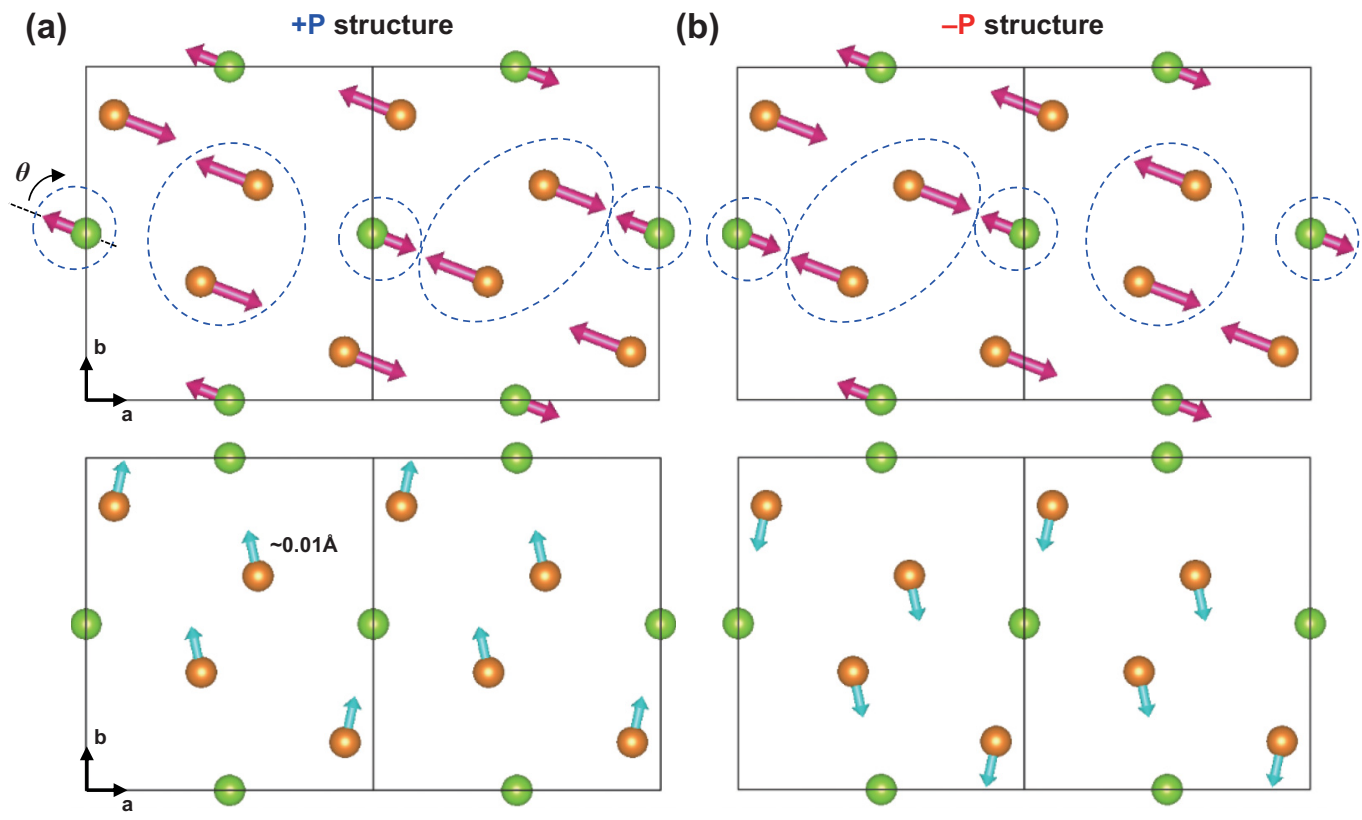

(c)

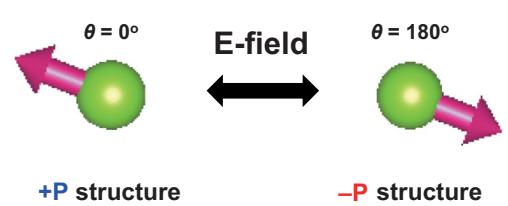

(d)

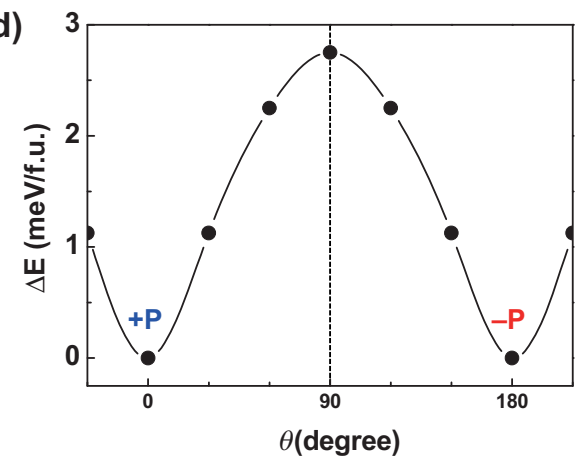

FIG. 2. (Color online) Spin structures and atomic displacement (ADP) vectors of (a) $+\mathrm{P}$ structure and (b) $-\mathrm{P}$ structure. Dashed circles in the upper panel of (a) and (b) denote the Mn-ion spin moments that reverse their directions in the course of the $+\mathrm{P} \leftrightarrow-\mathrm{P}$ polarization switching. The light blue arrows in the lower panel designate the ADP vectors. (c) Two degenerate spin orientations of the Mn ${ }^{4+}$ ion located at $(x, 0.5003, z)$. The $\mathrm{Mn}^{4+}$ spin moment with the canting angle $\theta=0^{\circ}$ in the $+\mathrm{P}$ structure is rotated by $180^{\circ}$ in the $-\mathrm{P}$ structure. (d) The computed Kohn-Sham energy plotted as a function of the canting angle $\theta$, showing the two degenerate spin states, $+\mathrm{P}$ and $-\mathrm{P}$.

Since the calculated ADP of $\mathrm{Mn}^{3+}$ is twice as large as that of $\mathrm{Mn}^{4+}\left(\mathrm{ADP}_{\mathrm{Mn} 3+}: \sim 0.01 \AA\right.$ and $\left.\mathrm{ADP}_{\mathrm{Mn} 4+}: \sim 0.005 \AA\right)$, we only present the ADP of $\mathrm{Mn}^{3+}$ ions for simplicity. According to our detailed $a b$ initio calculations, the ADP of $\mathrm{Mn}^{3+}$ ion, rather than those of $\mathrm{Mn}^{4+}$ and oxygen ions, plays a major role in the development of $P_{s}$ in $o$-TMO. In the $-\mathrm{P}$ structure, on the contrary, the net direction of the ADP of $\mathrm{Mn}^{3+}$ ions is reversed and thus is antiparallel to the $(+) b$ axis [Fig. 2(b)]. The spin configuration for the $-\mathrm{P}$ structure [upper panel of Fig. 2(b)] is equivalent to the spin configuration " $g$ '" in Ref. [12].

During the $+\mathrm{P} \leftrightarrow-\mathrm{P}$ polarization switching, the three distinct Mn spins located at $(x, 0.5003, z),\left(x^{\prime}, 0.3470, z^{\prime}\right)$, and $\left(x^{\prime \prime}, 0.6509, z^{\prime \prime}\right)$ are reversed in their directions. For example, the $\mathrm{Mn}^{4+}$ spin moment with the canting angle $\theta=0^{\circ}$ in the $+\mathrm{P}$ structure is rotated by $180^{\circ}$ in the $-\mathrm{P}$ structure [Fig. 2(c)]. Owing to this partial spin reversal associated with the $+\mathrm{P} \leftrightarrow-\mathrm{P}$ polarization switching, $(\downarrow \downarrow \uparrow \downarrow)$-type spin ordering along the $b$ axis in the $+\mathrm{P}$ structure undergoes a transition to $(\downarrow \uparrow \downarrow \downarrow)$-type spin ordering in the $-\mathrm{P}$ structure.
This indicates that the polarization switching is closely coupled with the exchange-striction coupling of a $\left(\boldsymbol{S}_{\boldsymbol{i}} \cdot \boldsymbol{S}_{\boldsymbol{j}}\right)$ type. This coupling term is indeed introduced in our previous discussion of the improper polarization, i.e., $\pm \gamma_{i}\left(S_{i}^{o} S_{j}^{o}\right) P$. According to our DFT calculations, the energy difference between the ferroelectric $\left(\theta=0^{\circ}\right.$ or $\left.180^{\circ}\right)$ and paraelectric $\left(\theta=90^{\circ}\right)$ states is $2.7 \mathrm{meV}$ per formula unit [Fig. 2(d)]. This barrier height is comparable to that of orthorhombic $\mathrm{YMnO}_{3}$ [21].

Before discussing the magnetic-field-induced polarization switching and associated spin structure, we have theoretically deduced a critical magnetic $(H)$ field needed for the polarization switching by considering the Landau free-energy density. The magnetic and magnetoelectric contributions to the free-energy density under $H_{x}$ field (along the $a$ axis) can be written as $\Phi_{m}=\left\{\frac{1}{2} \mu_{o} M_{x}^{2}-H_{x} M_{x}\right\}+v_{e}\left(S_{i}^{o} S_{j}^{o}\right) M_{x}^{2} P_{y}$, where $v_{e}$ is the extrinsic ME coupling constant, $P_{y}$ denotes the polarization developed along the $b$ axis of $P b 2_{1} m$, and $M_{x}$ designates the $x$ component of magnetization (along the $a$ axis). Then, the total free-energy density $(\Phi)$ of the $o$-TMO 
(a)

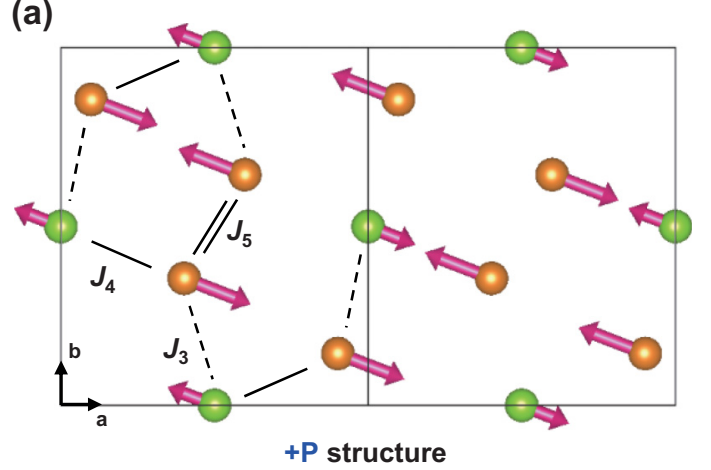

(c)

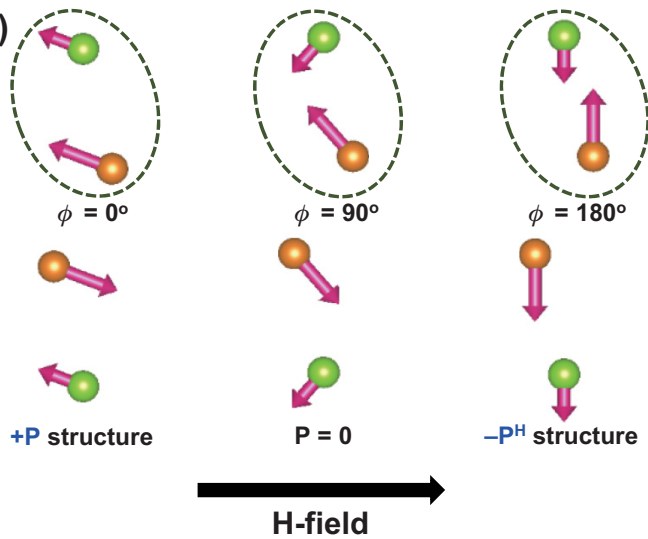

(b)

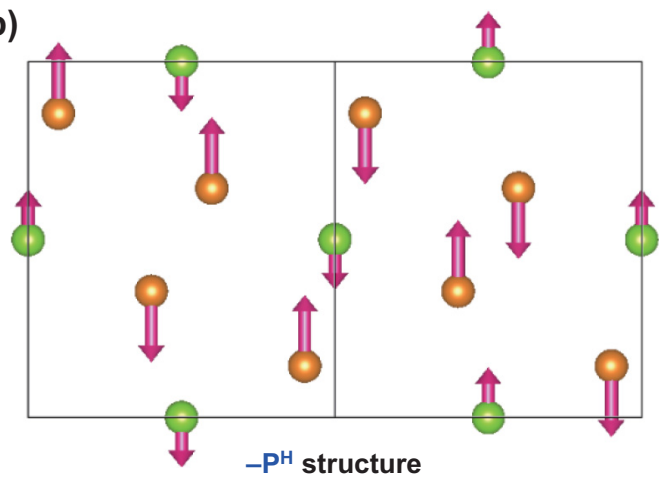

(d)

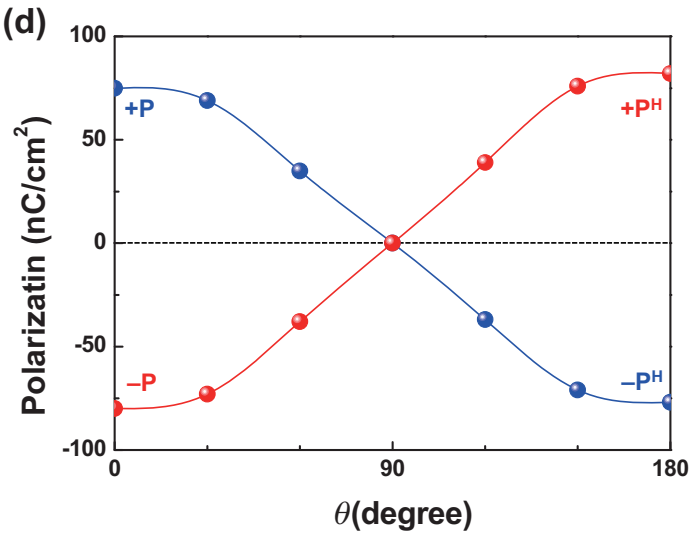

FIG. 3. (Color online) Spin configurations of (a) $+\mathrm{P}$ structure and (b) magnetic-field-induced $-\mathrm{P}^{H}$ structure projected on the $a-b$ plane. The three exchange parameters, $J_{3}, J_{4}$, and $J_{5}$, are explained in the text. On the other hand, $J_{1}$ and $J_{2}$ couple Mn ${ }^{4+}$ ions along the $c$ axis [not shown in (a)]. (c) The spin angle $\phi$ at three representative cases. The angle between the neighboring $\mathrm{Mn}^{3+}$ and $\mathrm{Mn}^{4+}$ spin moments along the $b$ axis (in the dashed circle) is defined by $\phi$. (d) The computed Berry-phase polarization of $o$-TMO, $P_{y}^{ \pm}\left(H_{x}\right)$, plotted as a function of $\phi$.

can be written as [22]

$$
\begin{aligned}
\Phi= & \Phi_{e}+\Phi_{m} \\
= & \frac{P_{y}^{2}}{2 \varepsilon_{o}} \pm \gamma_{i}\left(S_{i}^{o} S_{j}^{o}\right) P_{y}+\left\{\frac{1}{2} \mu_{o} M_{x}^{2}-H_{x} M_{x}\right\} \\
& +v_{e}\left(S_{i}^{o} S_{j}^{o}\right) M_{x}^{2} P_{y},
\end{aligned}
$$

where $\varepsilon_{o}$ denotes the static dielectric permittivity tensor along the $b$ axis (i.e., $\varepsilon_{y y}$ ). Minimizing $\Phi$ with respect to $P_{y}$ and $M_{x}$ and eliminating $M_{x}$ from the resulting relations yield the following expression for the $H$-field-dependent polarization along the $b$ axis:

$$
P_{y}^{ \pm}\left(H_{x}\right)=P_{y}^{ \pm}(0) \pm v_{e} \varepsilon_{o}\left(S_{i}^{o} S_{j}^{o}\right)\left(H_{x}^{2} / \mu_{o}^{2}\right)
$$

where $P_{y}^{+}(0) \equiv P_{y}^{+}\left(H_{x}=0\right)=-\varepsilon_{o}\left(S_{i}^{o} S_{j}^{o}\right) \gamma_{i}=-P_{y}^{-}(0)>0$ since $\gamma_{i}<0$. Thus, for $v_{e}<0$ (i.e., thermodynamically favorable extrinsic ME coupling), the application of an external $H$ field along the $a$ axis enhances the negative contribution to $P_{y}^{+}$and the polarization consequently undergoes a switching at a certain critical $H$ field $\left(H_{c}\right)$. Similarly, applying $H$ field enhances the positive contribution to $P_{y}^{-}$and $P_{y}^{-}$undergoes a switching from the initial negative value of $\varepsilon_{o}\left(S_{i}^{o} S_{j}^{o}\right) \gamma_{i}$ to a positive value at $H_{c}$. From Eq. (2), one can readily obtain that $H_{c}^{2}=\left|\frac{\gamma_{i}}{v_{e}}\right| \mu_{o}^{2}$. Thus, the critical $H$-field strength is proportional to the square root of the ratio of the intrinsic coupling constant $\left(\gamma_{i}\right)$ to the extrinsic ME coupling constant $\left(v_{e}\right)$.
Having identified $H_{c}$ for the magnetic-field-induced polarization switching, let us now examine the spin-structure modulation actually occurring in the course of the polarization switching. As mentioned previously, the polarization of $o$-TMO is reversed by the bias $H$ field of $\sim 1 T$ along the $a$ axis [3]. To deduce a stable spin configuration under a bias $H$ field along the $a$ axis, let us first define three exchange parameters $\left(J_{i}\right)$ in the AF zigzag spin chain [10,12], which refers to a five-member frustrated $\mathrm{Mn}$-spin loop, $\mathrm{Mn}^{4+}-\mathrm{Mn}^{3+}-\mathrm{Mn}^{3+}-\mathrm{Mn}^{4+}-\mathrm{Mn}^{3+}$, in the $a b$ plane [Fig. 3(a)]: (i) $J_{3}$ for the interchain superexchange interaction between the $\mathrm{AF} \mathrm{Mn}^{4+}-\mathrm{Mn}^{3+}$ spin pair (a dashed line) through the pyramidal base corner, (ii) $J_{4}$ for the intrachain exchange interaction between the $\mathrm{Mn}^{4+}-\mathrm{Mn}^{3+}$ spin pair (a single solid line) through the pyramidal top, and (iii) $J_{5}$ for the intrachain exchange interaction between the $\mathrm{Mn}^{3+}-\mathrm{Mn}^{3+}$ spin pair (a double solid line) through the pyramidal base edge. According to detailed analysis via a Heisenberg model [12], these exchange parameters are all negative, (i.e., AFM type) and $\left|J_{4}\right|,\left|J_{5}\right| \gg$ $\left|J_{3}\right|$. Thus, the spins should couple via $J_{4}$ and $J_{5}$ antiferromagnetically in the stable magnetic structure, as shown in Fig. 3(a).

Considering the relative strength of $J_{3}, J_{4}$, and $J_{5}$, one would expect that the AFM Mn-Mn spin coupling via $J_{4}$ or $J_{5}$ remains unaltered even under the bias $H$ field while the AFM Mn-Mn spin interaction via $J_{3}$ may undergo a change to ferromagnetic (FM) coupling. Based on this reasoning, we have delineated the modulated spin configuration under the 


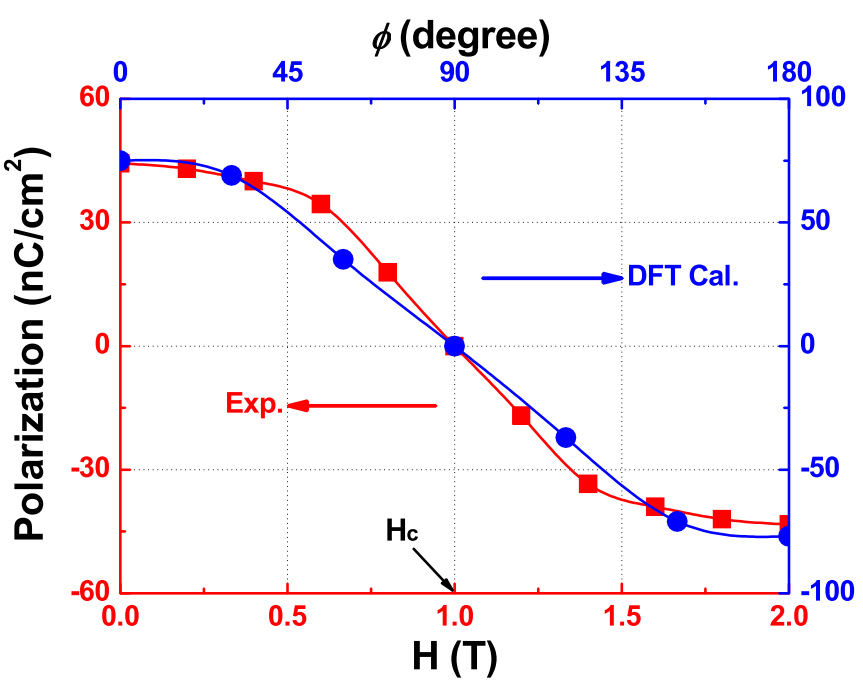

FIG. 4. (Color online) A comparison of the computed $\phi$ dependent polarization (denoted by blue circles) with the measured magnetic-field-dependent polarization (red squares) of $o$-TMO.

saturated bias $H$ field along the $a$ axis [Fig. 3(b)]. As denoted in Fig. 3(c), the degree of the modulation is represented by the spin angle, $\phi$, which is defined as the angle between the neighboring $\mathrm{Mn}^{4+}-\mathrm{Mn}^{3+}$ spin moments along the $b$ axis. The modulated spin structure represented by the extreme spin angle (i.e., $\phi=180^{\circ}$ ) is denoted by $-\mathrm{P}^{H}$ structure, and the corresponding spin configuration is shown in Fig. 3(b). This modulated spin structure is likely to occur under the saturated bias $H$ field along the $a$ axis and is similar to that of $\mathrm{BiMn}_{2} \mathrm{O}_{5}$ above the critical magnetic field, $H_{c}[23]$. $|\mathrm{P}|$ is expected to show its maximum at $\phi=0^{\circ}$ ( $+\mathrm{P}$ structure) or at $\phi=180^{\circ}\left(-\mathrm{P}^{H}\right.$ structure $)$ since $\left|\boldsymbol{S}_{\mathrm{Mn}^{4+}} \cdot \boldsymbol{S}_{\mathrm{Mn}^{3+}}\right|=1$ at these extreme angles. On the contrary, $|\mathrm{P}|$ is zero at $\phi=90^{\circ}$ since $\left|S_{\mathrm{Mn}^{4+}} \cdot \boldsymbol{S}_{\mathrm{Mn}^{3+}}\right|=0$ [Fig. 3(c)].

Figure 3(d) presents the computed polarization as a function of $\phi$, showing the polarization switching at $\phi=90^{\circ}$ (i.e., at $H_{c}$ ). The polarization which is initially $+\mathrm{P}$ decreases with increasing $\phi$ and eventually reaches a saturated negative value of $-\mathrm{P}^{H}$ at $\phi=180^{\circ}$. On the contrary, the polarization for the
-P structure (red line) shows a reverse trend [Fig. 3(d)]. Interestingly, we have shown that the computed polarization $\mathrm{P}(\phi)$ fairly well reproduces the measured $H$-field-dependent polarization $\mathrm{P}(H)$ at $3 \mathrm{~K}[3]$ by multiplying the DFT values with a scaling factor of $\{P(H=0) / P(\phi=0)\}$, as shown in Fig. 4 . In other words, both $\mathrm{P}(H)$ and $\left\{\frac{P(H=0)}{P(\phi=0)}\right\} P(\phi)$ are effectively described by a single master curve [Fig. 4]. Thus, the computed $\phi$-dependent DFT polarization satisfactorily accounts for the observed $H$-field-induced polarization switching. This clearly indicates that there exists 1:1 correspondence between $H$ and $\phi$ and, thus, the spin angle $\phi$ is a good measure of the bias $H$ field, $H_{x}$.

One can further deduce a numerical value of the critical magnetic-field strength $\left(H_{c}\right)$, starting from the relation that we have derived, i.e., $H_{c}^{2}=\left|\frac{\gamma_{i}}{v_{e}}\right| \mu_{o}^{2}$. Multiplying $\varepsilon_{o}\left(S_{i}^{o} S_{j}^{o}\right)$ to both denominator and numerator, one obtains

$$
H_{c}^{2}=\frac{\left|\gamma_{i}\right| \varepsilon_{o}\left(S_{i}^{o} S_{j}^{o}\right)}{\left|\nu_{e}\right| \varepsilon_{o}\left(S_{i}^{o} S_{j}^{o}\right)\left(\frac{1}{\mu_{o}^{2}}\right)}=\frac{P_{y}^{+}(0)}{|S|},
$$

where $S$ denotes the slope of $P_{y}^{+}\left(\right.$or $\left.P_{y}^{-}\right)$vs $H_{x}^{2}$ plot [See Eq. (2) for this]. In obtaining the last expression of Eq. (3), we used the previously derived relation that $P_{y}^{+}(0)=$ $\varepsilon_{o}\left(S_{i}^{o} S_{j}^{o}\right)\left|\gamma_{i}\right|$. According to Eq. (2), the magnitude of the slope is given by $|S|=\left|v_{e}\right| \varepsilon_{o}\left(S_{i}^{o} S_{j}^{o}\right) \mu_{o}^{-2}$. This justifies the last expression of Eq. (3). To deduce $H_{c}$, we have first estimated the slope of the $P_{y}^{+}$vs $H_{x}^{2}$ plot (not a $P_{y}^{+}$vs $H_{x}$ plot) using the $H$-field-dependent polarization data presented in Fig. 4 (filled red squares). It is $-40.9\left(\mathrm{nC} / \mathrm{cm}^{2} \mathrm{~T}^{2}\right)$. Since $P_{y}^{+}(0)=45\left(\mathrm{nC} / \mathrm{cm}^{2}\right)$ [Fig. 4], $H_{c}$ is estimated to be $\sim 1.05 \mathrm{~T}$, which nearly coincides with the experimental value of $\sim 1.0 \mathrm{~T}$ [Fig. 4].

Figure 5 illustrates the spatial distribution of the valenceelectron density by plotting the difference in the computed partial charge density $[\triangle \mathrm{PCD}(\mathbf{r})]$ between the ferroelectric $\left(\phi=0^{\circ}\right.$ or $\left.180^{\circ}\right)$ and paraelectric $\left(\phi=90^{\circ}\right)$ phases within the energy range between -0.55 and $0 \mathrm{eV}$ below the valence-band top (i.e., corresponding to the valence-bonding energy region). Thus, $\triangle \mathrm{PCD}(\mathbf{r})$ signifies the excess or deficient valenceelectron density associated with the para-to-ferroelectric phase transition. It is interesting to notice that the excess (deficient) (a)

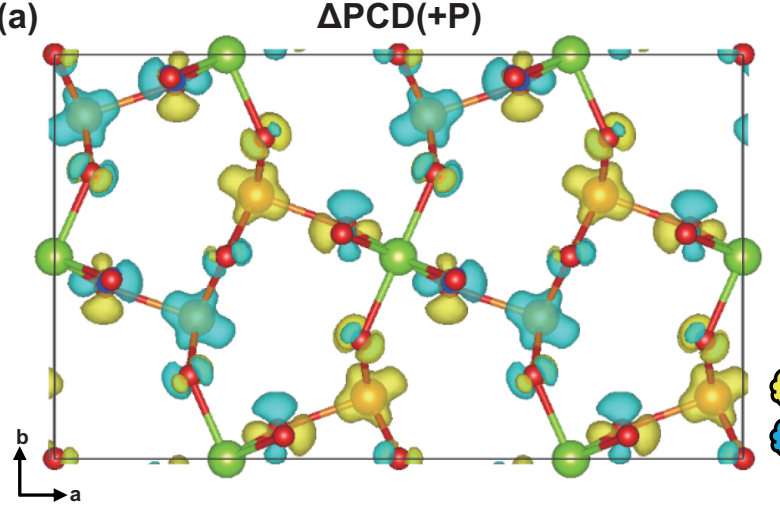

(b)

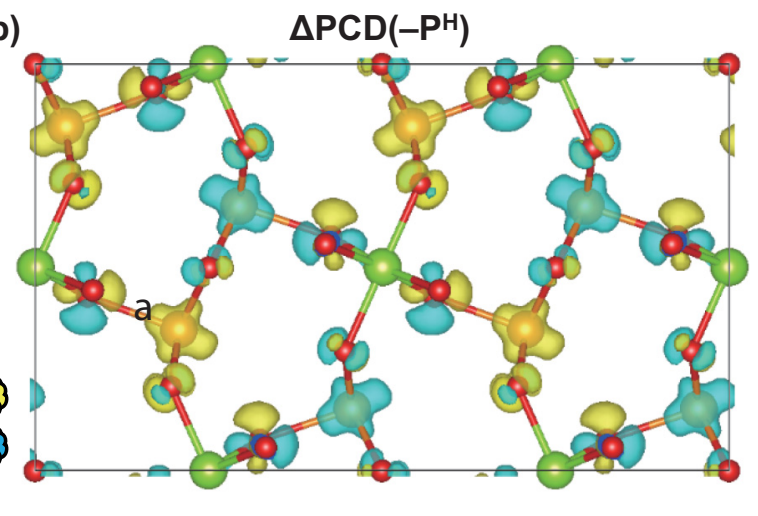

FIG. 5. (Color online) A comparison of the difference in the partial charge density $\left(\triangle \mathrm{PCD} \equiv \mathrm{PCD}_{\text {ferro }}-\mathrm{PCD}_{\text {para }}\right)$ contour of $(\mathrm{a})+\mathrm{P}$ structure and (b) $-\mathrm{P}^{H}$ structure within the energy range between -0.55 and $0 \mathrm{eV}$ below the valence-band top. Thus, $\triangle \mathrm{PCD}$ signifies the excess or deficient valence-electron density associated with the para-to-ferroelectric transition. The yellow and light blue electron clouds denote the excess and deficient electronic charge-density regions, respectively. The isosurface level is equal to $0.0002 e / \AA$. 

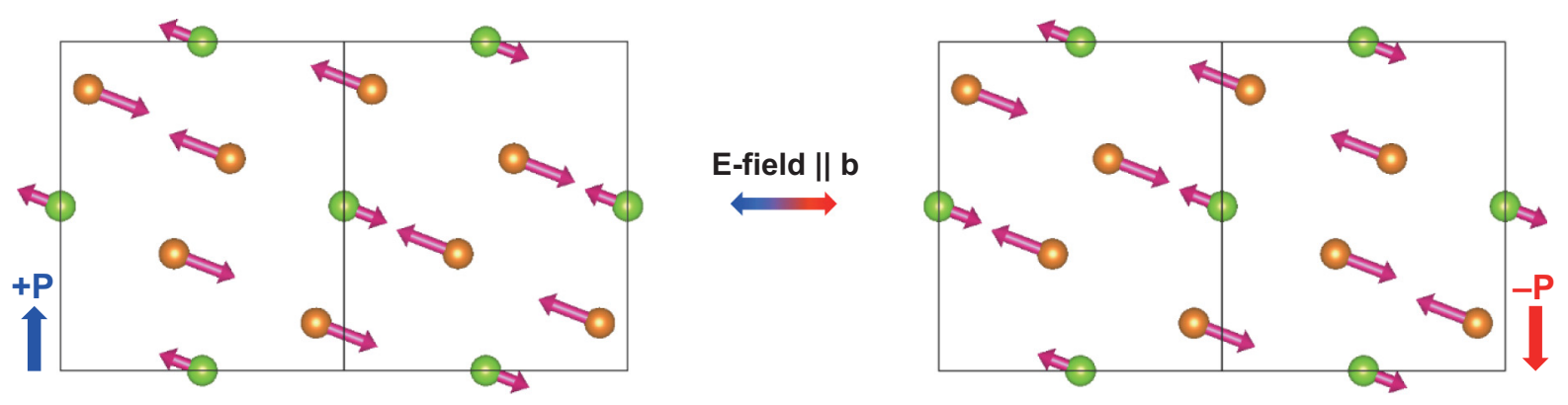

H-field || a

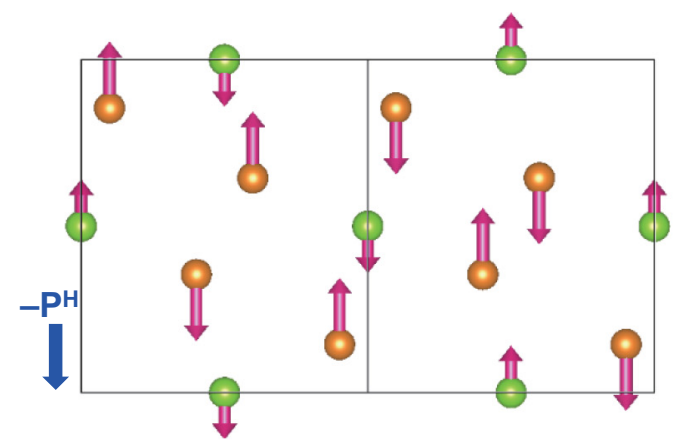

1

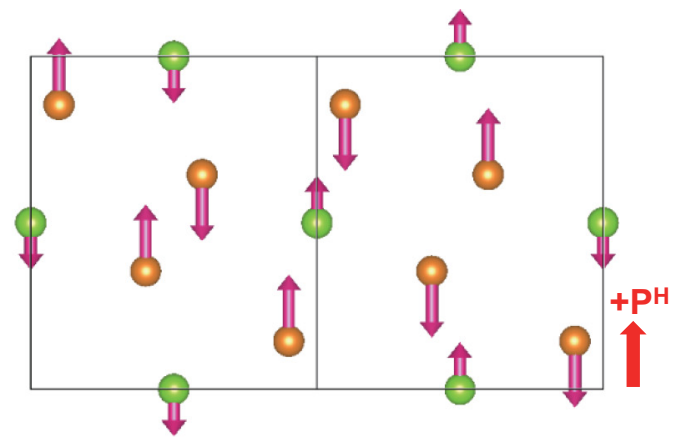

FIG. 6. (Color online) A pictorial representation of the spin configuration for the four distinct multiferroic states of $o$-TMO. The variation in the spin configuration associated with the electric-field-induced polarization switching is shown in the upper panel. On the other hand, the two distinct processes of the magnetic-field-induced polarization switching are represented vertically: $+\mathrm{P}-$ to- $-\mathrm{P}^{H}$ switching in the left-hand side column and $-\mathrm{P}$-to- $+\mathrm{P}^{H}$ switching in the right-hand side column.

valence-electron density becomes deficient (excess) for the $H$-field-induced change from the $+\mathrm{P}$ structure to the $-\mathrm{P}^{H}$ structure. These computed results of $\triangle \mathrm{PCD}$ demonstrate that like the ADPs of all constituting ions, the sign of the excess valence-electron density (but not the core-electron density) also undergoes a switching by the bias $H$ field.

In Fig. 6, we pictorially summarize the result of our first-principles study on the magnetically induced polarization switching. The degenerate $+\mathrm{P}$ and $-\mathrm{P}$ structures in the absence of $H$ field can be switched to each other by applying a bias electric $(E)$ field along the $b$ axis. On the other hand, the $+\mathrm{P}(-\mathrm{P})$ and $-\mathrm{P}^{H}\left(+\mathrm{P}^{H}\right)$ structures can be switched to each other by applying a bias $H$ field via the extrinsic ME coupling mechanism $\left[v_{e} \varepsilon_{o}\left(S_{i}^{o} S_{j}^{o}\right)\left(H_{x}^{2} / \mu_{o}^{2}\right)\right.$ term in Eq. (2)]. The switching between the $-\mathrm{P}^{H}$ and $+\mathrm{P}^{H}$ states by an external $E$ field is a purely computational prediction and, thus, needs future experimental demonstrations.

\section{CONCLUSIONS}

In conclusion, we have proposed a modulated spin structure responsible for the field-induced polarization switching by considering the relative strength of the three relevant exchange parameters. The proposed modulated structure under a bias $H_{x}$ field is described in terms of the spin angle $\phi$ between the neighboring $\mathrm{Mn}^{4+}-\mathrm{Mn}^{3+}$ spin moments. The computed polarization as a function of $\phi$ successfully accounts for the observed $H$-field-induced polarization switching in $o$-TMO. This conclusion was obtained by demonstrating 1:1 correspondence between $\phi$ and the bias magnetic-field strength, $H$. The computed partial charge density $(\triangle \mathrm{PCD})$ further demonstrates that the sign of the excess valence-electron density also undergoes a switching by the bias $H$ field along the $a$ axis.

\section{ACKNOWLEDGMENTS}

This work was financially supported by the National Research Foundation (NRF) Grants funded by the Korea Government (MSIP) (Grants No. 2012R1A1A2041628 and No. 2013R1A2A2A01068274). Computational resources provided by KISTI Super-computing Center (Project No. KSC2013-C3-067) are acknowledged.
[1] J. Wang, J. B. Neaton, H. Zheng, V. Nagarajan, S. B. Ogale, B. Liu, D. Viehland, V. Vaithyanathan, D. G. Scholom, U. V. Waghmare, N. A. Spaldin, K. M. Rabe, M. Wuttig, and R. Ramesh, Science 299, 1719 (2003).
[2] T. Kimura, T. Goto, H. Shintani, K. Ishizaka, T. Arima, and Y. Tokura, Nature 426, 55 (2003).

[3] N. Hur, S. Park, P. A. Sharma, J. S. Ahn, S. Guha, and S. W. Cheong, Nature 429, 392 (2004). 
[4] T. Lottermoser, T. Lonkai, U. Amann, D. Hohlwein, J. Ihringer, and M. Fiebig, Nature 430, 541 (2004).

[5] W. Eerenstein, N. D. Mathur, and J. F. Scott, Nature 442, 759 (2006).

[6] S.-W. Cheong and M. Mostovoy, Nat. Mater. 6, 13 (2007).

[7] F. Kagawa, M. Mochizuki, Y. Onose, H. Murakawa, Y. Kaneko, N. Furukawa, and Y. Tokura, Phys. Rev. Lett. 102, 057604 (2009).

[8] B. Lorenz, Y.-Q. Wang, and C.-W. Chu, Phys. Rev. B 76, 104405 (2007).

[9] S. Ishiwata, Y. Kaneko, Y. Tokunaga, Y. Taguchi, T. H. Arima, and Y. Tokura, Phys. Rev. B 81, 100411(R) (2010).

[10] L. C. Chapon, G. R. Blake, M. J. Gutmann, S. Park, N. Hur, P. G. Radaelli, and S.-W. Cheong, Phys. Rev. Lett. 93, 177402 (2004).

[11] C. Wang, G.-C. Guo, and L. He, Phys. Rev. Lett. 99, 177202 (2007).

[12] C. Wang, G.-C. Guo, and L. He, Phys. Rev. B 77, 134113 (2008).

[13] K. Cao, G.-C. Guo, D. Vanderbilt, and L. He, Phys. Rev. Lett. 103, 257201 (2009).
[14] T.-R. Chang, H.-T. Jeng, C.-Y. Ren, and C.-S. Hsue, Phys. Rev. B 84, 024421 (2011).

[15] G. Kresse and D. Joubert, Phys. Rev. B 59, 1758 (1999).

[16] G. Kresse and J. Furthmüller, Phys. Rev. B 54, 11169 (1996).

[17] R. D. King-Smith and D. Vanderbilt, Phys. Rev. B 47, 1651 (1993); D. Vanderbilt and R. D. King-Smith, ibid. 48, 4442 (1993).

[18] P. E. Blöchl, O. Jepsen, and O. K. Andersen, Phys. Rev. B 49 16223 (1994).

[19] G. Giovannetti and J. van den Brink, Phys. Rev. Lett. 100, 227603 (2008)

[20] J. A. Alonso, M. T. Casais, M. M Martínez-Lope, J. L. Martínez, and M. T. Fernández-Díaz, J. Phys.: Condens. Matter 9, 8515 (1997).

[21] J.-H. Lee, S. Song, and H. M. Jang, Phys. Rev. B 88, 014116 (2013).

[22] P. Tolédano, W. Schranz, and G. Krexner, Phys. Rev. B 79, 144103 (2009).

[23] J. W. Kim, S. Y. Haam, Y. S. Oh, S. Park, S.-W. Cheong, P. A. Sharma, M. Jaime, N. Harrison, J. H. Han, G.-S. Jeon, P. Coleman, and K. H. Kim, Proc. Natl. Acad. Sci. USA 106, 15573 (2009) 